\title{
The impact of spore surface attributes of Lichtheimia corymbifera during infection of human cells
}

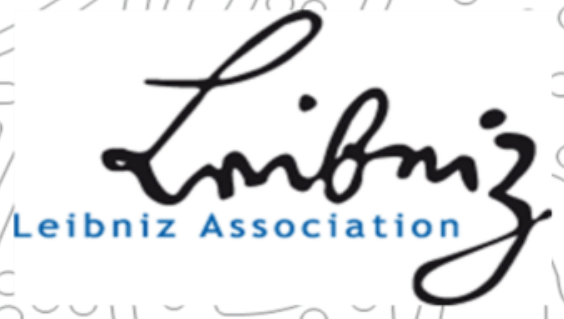
HK

\section{Dolly Montano ${ }^{1,2}$, Volker U. Schwartze ${ }^{1,2}$, Mohamed Hassan ${ }^{1,2}$, Hans-Martin Dahse ${ }^{2}$, Kerstin Voigt ${ }^{1,2}$.}

[1] Friedrich-Schiller University, Faculty of Biological Sciences, Institute of Microbiology. Jena, Germany.

[2] Leibniz Institute for Natural Product Research and Infection Biology - Hans Knöll Institute. Jena, Germany.

\section{Introduction}

Lichtheimia corymbifera is a causative agent of mucormycosis, a life-threatening infection caused by members of the fungal order Mucorales. This pathogen can invade blood vessels of immunocompromised patients, leading to infarction, thrombosis and tissue necrosis [1]. Currently, therapeutic options against mucormycosis are limited due to difficult earlydiagnosis, antifungal drug resistance and lower number of studies related to host-pathogen interactions.

\section{Scientific background}

- Spore cell-wall proteins and pigments are known to play an important role in other fungal pathogens when interacting with host cells ${ }^{[2]}$.

- The full genome sequences of $L$. corymbifera provides a new molecular basis to study pathogenicity-associated features [3].

- Current studies in our group predict genes encoding proteins involved in host-pathogen interactions.

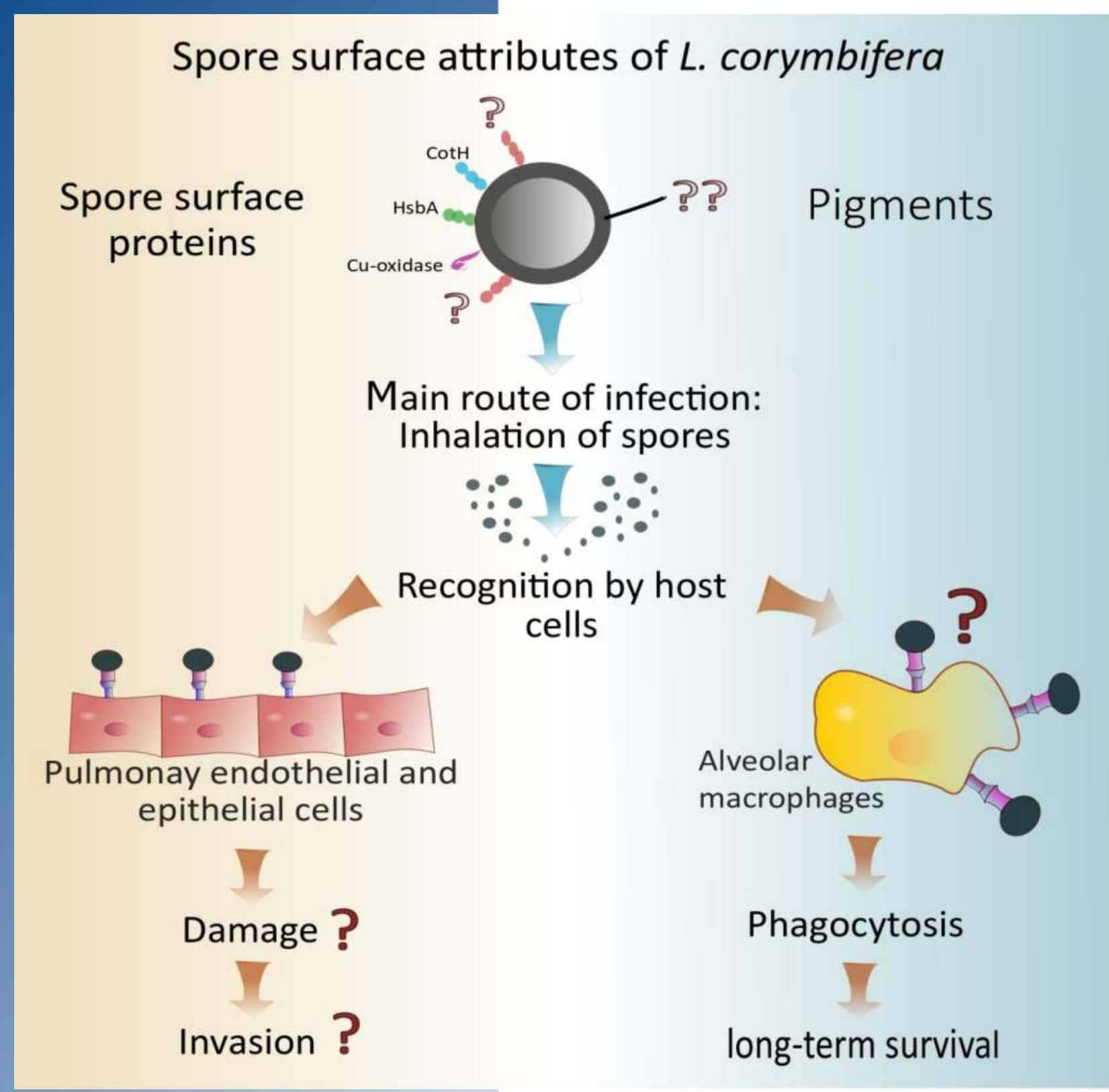

Research Question

How do spore surface proteins and pigments of L. corymbifera contribute to the infection of human host cells?

\section{Work packages}

Establish in vitro infection models with nonsurface proteins in order to block alveolar macrophages, the first barrier to pulmonary infections.

Determine host-pathogen interactions during initial stages of infection

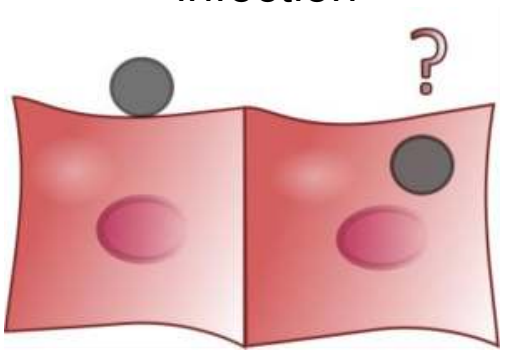

Methods: fluorescence staining, laser scanning microscopy, and flow cytometric analysis.

Evaluate the long-term survival of spores with different pigment phenotypes.
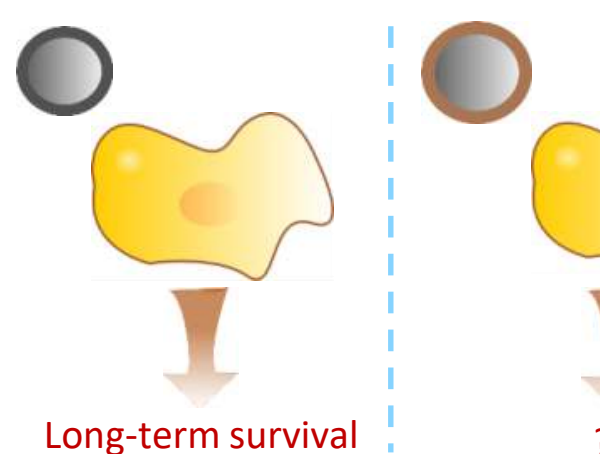

Determine the contribution of spore pigments in the long-term survival

Methods: laser scanning microscopy, flow cytometric analysis, and apoptosis assay.
Generate specific antibodies gand-receptor interactions.

Screening-system for detection of relevant proteins involved in recognition by host cells
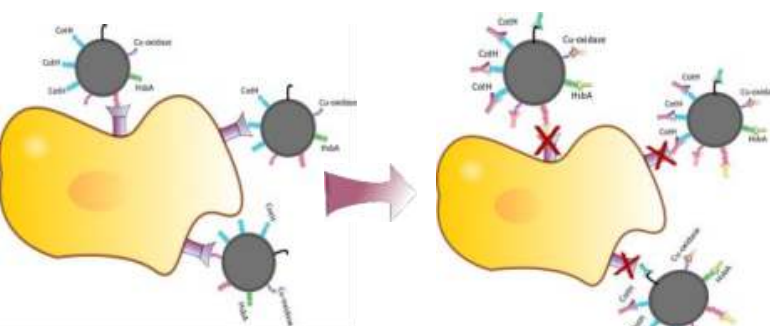

Methods: generation of antibodies yeast-surface-display system, protein purification, and phagocytosis assays.

Silence genes involved in the initial host-pathogen interactions and employ yeast surface display as a gain-offunction system.

Confirm the role of relevant spore surface proteins

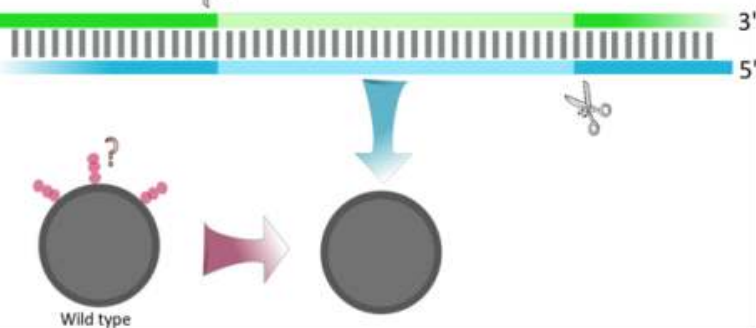

Methods: genome engineering with CRISPR, and yeast-surface-display.

\section{Outlook}

With all these strategies we expect to determine a set of spore surface proteins that play an important role during recognition by human cells. Furthermore, we hope to disclose whether pigments of the fungus contribute to its long-term survival inside macrophages, which could provide us insights to develop novel diagnostics and therapeutic strategies to counteract infections caused by $L$. corymbifera.

Acknowledgment to

- Hans Knöll Institute: Jena Microbial Resource Collection (JMRC), Infection Biology, and Molecular and Applied Microbiology. Jena-Germany.

- Friedrich-Schiller University, JMRC. Jena-Germany.

- $\quad$ CRC/TR 124 FungiNet (TP A6, C6) funding of this project.

\section{References}

[1] Sugar AM. 1992. Mucormycosis. Clin Infec Dis. 14: 126-129

[2] Heinekamp T, et al. 2013. Aspergillus fumigatus melanins: interference with the host endocytosis pathway and impact on virulence. Front Microbiol. 18;3:440.

[3] Schwartze V, et al. 2014. Gene Expansion Shapes Genome Architecture in the Human Pathogen Lichtheimia corymbifera: An Evolutionary Genomics Analysis in the Ancient Terrestrial Mucorales 